\title{
Pharyngo-jugular fistula after "salvage" total laryngectomy: a case report
}

Erika Crosetti ${ }^{*}$, Andrea Fulcheri $^{2}$ and Giovanni Succo ${ }^{2}$

\begin{abstract}
Introduction: We present a rare case of pharyngo-jugular fistula in a patient who underwent salvage total laryngectomy after organ-sparing radiochemotherapy.

Case presentation: A 77-year-old Caucasian man underwent total laryngectomy and bilateral neck dissection as salvage surgery after the failure of radiochemotherapy at another hospital. Thirty-five days after surgery, he was admitted to our emergency room for fever and massive oral bleeding during meals. Videopanendoscopy showed the presence of a large clot at the base of his tongue, while a neck computed tomography scan showed a pharyngo-jugular fistula with the presence of air in the left internal jugular vein. Cervicotomy was performed: the internal jugular vein was ligated and sectioned, and the pharyngeal defect was repaired with a pectoralis major myocutaneous flap. The postoperative period was uneventful. Twenty-five days post surgery, videofluorography showed the fistula had disappeared. Our patient then began oral feeding without complications and was discharged. At present, 5 years after the operation, our patient is alive and shows no evidence of disease.
\end{abstract}

Conclusions: Pharyngo-jugular fistula is an uncommon complication after total laryngectomy, especially in the chemoradiation era, which is potentially fatal if not promptly treated.

Keywords: Pharyngo-jugular fistula, Fistula, Complication after total laryngectomy

\section{Introduction}

Diagnosis and correct management of postoperative complications after head and neck oncological surgery is of substantial interest given the complexity of potential clinical conditions and associated morbidity, with an increase in the duration of hospitalization and related costs. The increasingly widespread use of organ-sparing radiochemotherapy protocols has led to the use of salvage surgery only in cases of persistent or recurrent disease [1], which is, however, associated with a greater incidence of major complications compared to planned posttreatment neck dissection (24-61\%) [1-3]. In salvage total laryngectomy, the most frequent early complications are pharyngocutaneous fistula, wound dehiscence and postoperative hemorrhage; common late complications include dysphagia and stenosis of the tracheostomy [4].

In this case report, we present a rare case of pharyngojugular fistula in a patient who underwent salvage total laryngectomy after organ-sparing radiochemotherapy.

\footnotetext{
* Correspondence: erikacro73@yahoo.com

'ENT Department, Martini Hospital, Via Tofane 71, 10141 Turin, Italy

Full list of author information is available at the end of the article
}

\section{Case presentation}

A 77-year-old Caucasian man was admitted to our department, complaining of two episodes of massive oral hemorrhage during meals, accompanied by odynophagia and fever. Thirty-five days before, in another hospital, our patient had undergone total laryngectomy and bilateral selective neck dissection after failure of organsparing salvage radiochemotherapy (cisplatin $100 \mathrm{mg} / \mathrm{m}^{2}$ every 21 days for three cycles, and standard radiotherapy: $70 \mathrm{~Gy}, 1.8 \mathrm{~Gy} /$ day). The postoperative period was complicated by a small pharyngocutaneous fistula, which resolved in a few days with compressive bandages.

Videoendoscopy demonstrated the presence of a large clot at the base of his tongue. A neck computed tomography (CT) scan (Figs. 1 and 2) revealed a pharyngojugular fistula with the presence of air in the left internal jugular vein (IJV). Our patient was subjected to an emergency operation consisting in cervicotomy, ligation and section of the left IJV and closure of the fistula using a pectoralis major myocutaneous flap.

The postoperative period was uneventful. Twenty-five days post surgery, videofluorography was performed, 


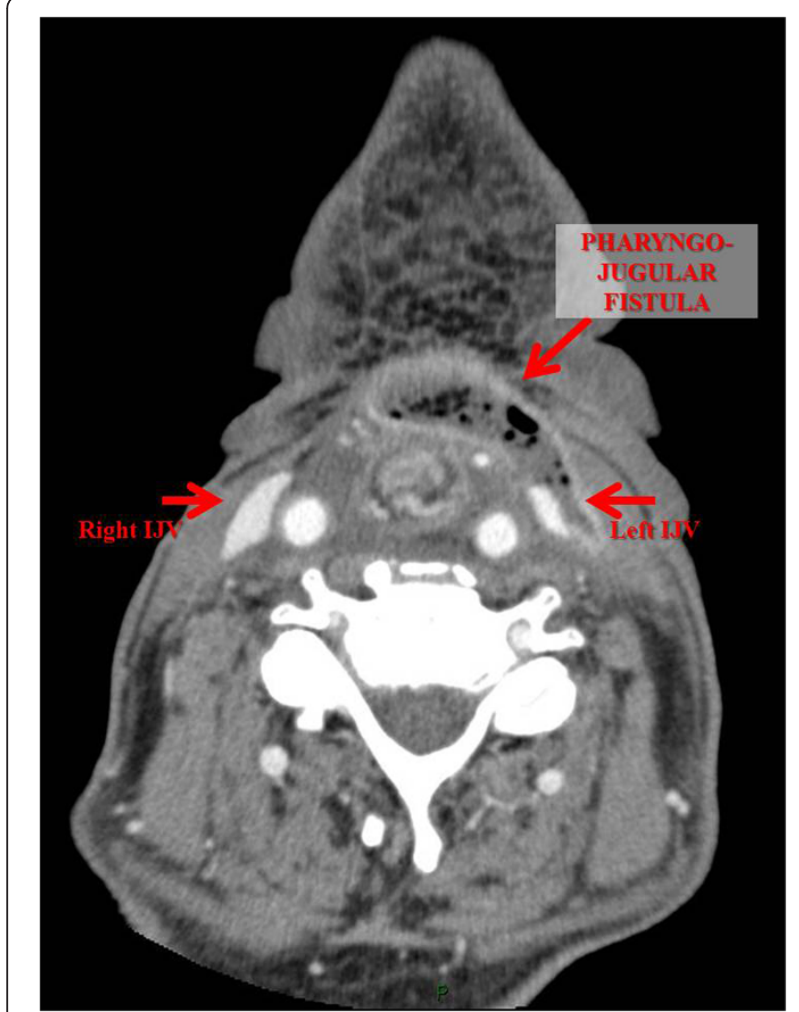

Fig. 1 Neck computed tomography (axial view) showed the presence of a fistula between the left internal jugular vein and pharynx

which showed the fistula had disappeared. Our patient began oral feeding and was discharged. At present, 5 years after the operation, our patient is alive and shows no evidence of disease.

\section{Discussion}

Salvage surgery of the neck and upper aerodigestive tract in the era of chemoradiation is unfortunately associated with a greater incidence of major postoperative complications compared to primary surgery, with an increase in morbidity, mortality, duration of hospitalization and related costs [4-8]. In a recent article, in 24 patients previously treated with chemoradiation and subjected to total laryngectomy combined with total or partial pharyngectomy, Sewnaik et al. [3] reported a postoperative complication rate of $92 \%$. Among early complications (within 3 months of operation), the most common were pharyngocutaneous fistula (50\%), wound dehiscence (29\%) and postoperative hemorrhage (21\%), while among late complications dysphagia (25\%) and stenosis of the tracheostoma were most frequent (21\%).

In the literature, the reported rate of pharyngocutaneous fistula following total laryngectomy is around 16\% after primary surgery [9], which increases to $19-50 \%$ after salvage laryngectomy $[3,10,11]$. Several authors have investigated the risk factors for pharyngocutaneous fistula after

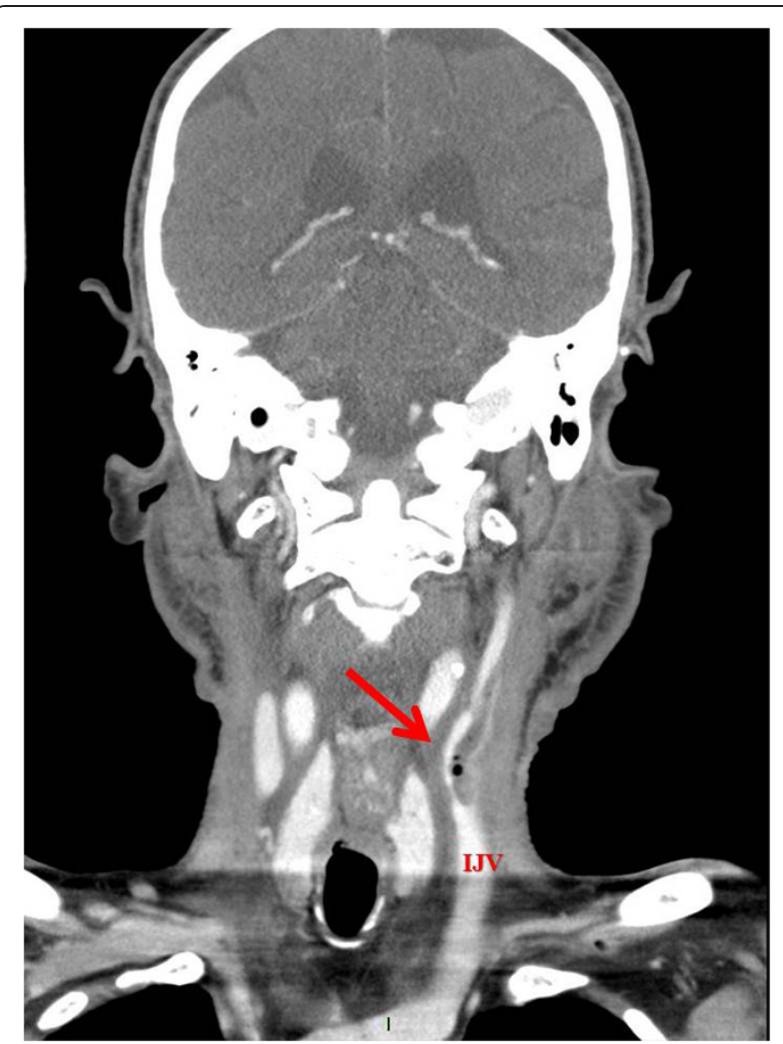

Fig. 2 Neck computed tomography (coronal view) revealed a fistula between the left internal jugular vein and pharynx

salvage laryngectomy, which include advanced stage of primary tumor [11], infection caused by methicillinresistant Staphylococcus aureus [12] and previous radiotherapy [11].

Lee et al. [13] evaluated the risk factors for surgical site infection in patients undergoing major head and neck oncologic surgery. Infections at the site of operation, defined as infection of the surgical wound within 30 days following surgery and manifested as a purulent exudate or orocutaneous/pharyngocutaneous fistula, were observed in $10-45 \%$ of patients. Among the risk factors identified, there were both patient-related (co-morbidity, body mass index [BMI], tobacco use, alcoholism, American Society of Anesthesiologists [ASA] grade) and disease-related factors (site and stage of tumor), in addition to iatrogenic factors, such as previous radiochemotherapy, presence of tracheostoma, clean-contaminated wounds and extent of neck dissection [4, 6-8].

Morgan et al. [14], in a retrospective analysis of 38 patients subjected to salvage surgery after failure of organsparing radiochemotherapy for advanced head and neck tumors, reported the presence of pharyngocutaneous fistula in $20 \%$ of cases in whom pharyngotomy with successive pharyngeal suture was performed, and a single case of chylous fistula with carotid artery exposure in a 
patient subjected to planned neck dissection. As can be reasonably expected, the complication rate was lower in the group of patients undergoing neck dissection only compared to those subjected to neck dissection plus intervention at the primary tumor site, which also differed according to the type of wound complication ( $4 \%$ in the case of clean wounds, $23 \%$ for clean-contaminated).

Recently, Paydarfar et al. [15] carried out a systematic literature review on the risk factors for pharyngocutaneous fistula, which confirmed that the relative risk (RR) of developing a fistula after salvage laryngectomy was significantly higher after radiotherapy (RR 2.28 [1.59-3.25]) or radiotherapy associated with neck dissection (RR 2.96 [1.51-5.80]). Other risk factors included a reduced level of postoperative hemoglobin $(<12.5 \mathrm{~g} / \mathrm{dL}, \mathrm{RR} 2.10$ [1.24-3.55]) and previous tracheostomy (RR 1.60 [1.05-2.44]). The severity and mean time of healing of a pharyngocutaneous fistula have been found to be significantly greater in patients with prior radiochemotherapy compared to those without preoperative radiochemotherapy [15].

To the best of our knowledge, this is the first report of a pharyngo-jugular fistula. In the present case, the postoperative period after the primary operation (total laryngectomy and bilateral selective neck dissection) was complicated by a pharyngocutaneous fistula that resolved with compressive bandages. It is possible that the bandage led to deviation and successive progression of the fistula toward the lateral cervical region, favoring pharyngocutaneous healing but with the formation of a pharyngo-jugular fistula.

In 2003, Cleland-Zamudio, in describing six cases of rupture of the IJV, observed a temporal relationship between rupture of the vessel and pharyngocutaneous fistula: the formation of the fistula generally preceded rupture of the vein [9].

In our opinion, a pharyngocutaneous fistula following salvage laryngectomy due to failure of an organ-sparing protocol is a common complication that can be resolved with either local dressing or reconstructive surgery. After apparent resolution of the fistula, it is important to perform videofluorography until it is completely resolved; the examination should always be carried out prior to reinitiating oral feeding. The use of pedicled or free flaps for reconstruction or the simple coverage of cervical vessels are valid surgical options in the attempt to reduce postoperative complications such as pharyngocutaneous and pharyngo-jugular fistula, with obvious benefits in terms of duration of hospitalization and associated costs.

\section{Conclusions}

The increasingly widespread use of organ-sparing radiochemotherapy protocols has led to an increase in salvage surgical procedures in patients with persistent or recurrent disease [1], which are associated with a higher rate of complications compared to primary surgery [2, 3, 5-7]. Among these, pharyngocutaneous fistula is the most common.

The case described herein of a nonfatal pharyngojugular fistula is, to the best of our knowledge, the first such case described in the literature: this is a very rare complication after total laryngectomy, especially after salvage surgery, and is potentially fatal if not treated promptly.

\section{Consent}

Written informed consent was obtained from the patient for publication of this manuscript and any accompanying images. A copy of the written consent is available for review by the Editor-in-Chief of this journal.

\section{Abbreviations}

BMI: Body mass index; CT: Computed tomography; IVG: Internal jugular vein; RR: Relative risk.

\section{Competing interests}

The authors declare that they have no competing interests.

\section{Authors' contributions}

AF visited the patient, diagnosed the complication, analyzed and interpreted the clinical data, and contributed to drafting the manuscript. GS performed the operation, and was involved in the conception and critical review of the manuscript. EC performed the operation, regularly visited the patient, and was involved in the conception and drafting of the manuscript. All authors read and approved the final manuscript.

\section{Author details}

${ }^{1}$ ENT Department, Martini Hospital, Via Tofane 71, 10141 Turin, Italy. ${ }^{2}$ ENT Department, S. Luigi Gonzaga Hospital, University of Turin, Regione Gonzole, 10, 10043 Orbassano, Italy.

Received: 5 July 2015 Accepted: 15 September 2015

Published online: 06 October 2015

\section{References}

1. Boyd TS, Harari PM, Tannehill SP, Voytovich MC, Hartig GK, Ford CN, et al. Planned postradiotherapy neck dissections in patients with advanced head and neck cancer. Head Neck. 1998;20:132-7.

2. Davidson BJ, Newkirk KA, Harter KW, Picken CA, Cullen KJ, Sessions RB. Complications from planned, post-treatment neck dissections. Arch Otolaryngol Head Neck Surg. 1999;125:401-5.

3. Sewnaik A, Keereweer S, Al-Mamgani A, Baatenburg de Jong RJ, Wieringa $\mathrm{MH}$, Meeuwis CA, et al. High complication risk of salvage surgery after chemo-radiation failures. Acta Otolaryngol. 2012;132:96-100.

4. Lotfi CJ, Cavalcanti Rde C, Costa E, Silva AM, Latorre Mdo R, Ribeiro Kde C, et al. Risk factors for surgical-site infections in head and neck surgery. Otolaryngol Head Neck Surg. 2008;138:74-80.

5. Girod DA, McCulloch TM, Tsue TT, Weymuller Jr EA. Risk factors for complications in clean-contaminated head and neck surgical procedures. Head Neck. 1995;17:7-13.

6. de Melo GM, Ribeiro KC, Kowalski LP, Deheinzelin D. Risk factors for postoperative complications in oral cancer and their prognostic implications. Arch Otolaryngol Head Neck Surg. 2001;127:828-33.

7. Penel N, Fournier C, Lefebvre D, Lefebvre JL. Multivariate analysis of risk factors for wound infection in head and neck squamous cell carcinoma surgery with opening of mucosa. Study of 260 surgical procedures. Oral Oncol. 2005;41:294-303.

8. Ogihara H, Takeuchi K, Majima Y. Risk factors of postoperative infection in head and neck surgery. Auris Nasus Larynx. 2009;36:457-60. 
9. Cleland-Zamudio SS, Wax MK, Smith JD, Cohen Jl. Ruptured internal jugular vein: a postoperative complication of modified/selected neck dissection. Head Neck. 2003;25:357-60

10. Dirven R, Swinson BD, Gao K, Clark JR. The assessment of pharyngocutaneous fistula rate in patients treated primarily with definitive radiotherapy followed by salvage surgery of the larynx and hypopharynx. Laryngoscope. 2009;119:1691-95.

11. Grau C, Johansen LV, Hansen HS, Andersen E, Godballe C, Andersen LJ, et al. Salvage laryngectomy and pharyngo-cutaneous fistulae after primary radiotherapy for head and neck cancer: a national survey from DAHANCA. Head Neck. 2003;25:711-6.

12. Jeannon JP, Orabi A, Manganarsi A, Simo R. Methicillin resistant Staphylococcus Aureus infection as a causative agent of fistula formation following total laryngectomy for advanced head and neck cancer. Head Neck Oncol. 2010;2:14

13. Lee DH, Kim SY, Nam SY, Choi SH, Choi JW, Roh JL. Risk factors of surgical site infection in patients undergoing major oncological surgery for head and neck cancer. Oral Oncol. 2011;47:528-31.

14. Morgan JE, Breau RL, Suen JS, Hanna EY. Surgical wound complications after intensive chemo-radiotherapy for advanced squamous cell carcinoma of the head and neck. Arch Otolaryngol Head Neck Surg. 2007;133:10-4.

15. Paydarfar JA, Birkmeyer NJ. Complications in head and neck surgery: a meta-analysis of postlaryngectomy pharyngocutaneous fistula. Arch Otolaryngol Head Neck Surg. 2006;132:67-72.

\section{Submit your next manuscript to BioMed Central and take full advantage of:}

- Convenient online submission

- Thorough peer review

- No space constraints or color figure charges

- Immediate publication on acceptance

- Inclusion in PubMed, CAS, Scopus and Google Scholar

- Research which is freely available for redistribution 